Published in final edited form as:

Schizophr Res. 2013 August ; 148(0): 126-129. doi:10.1016/j.schres.2013.05.006.

\title{
Drug and alcohol trajectories among adults with schizophrenia: Data from the CATIE Study
}

\author{
Richard A. Van Dorn a, ${ }^{*}$, Sarah L. Desmarais ${ }^{b}$, Stephen J. Tuellera, Jennifer M. Jolleyc, \\ Kiersten L. Johnson ${ }^{b}$, and Marvin S. Swartz ${ }^{d}$ \\ Richard A. Van Dorn: rvandorn@rti.org; Sarah L. Desmarais: sdesmarais@ncsu.edu; Stephen J. Tueller: stueller@rti.org; \\ Jennifer M. Jolley: jmjolley@email.unc.edu; Kiersten L. Johnson: kljeske@ncsu.edu; Marvin S. Swartz: \\ marvin.swartz@duke.edu

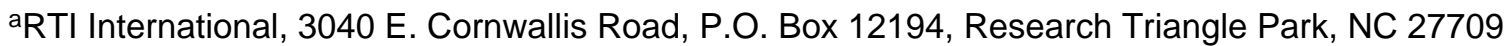 \\ bDepartment of Psychology, North Carolina State University, Campus Box 7650, Raleigh, NC \\ 27695
}

${ }^{\circ}$ Cecil G. Sheps Center for Health Services Research, University of North Carolina at Chapel Hill, 725 Martin Luther King, Jr. Blvd, Campus Box 7590, Chapel Hill, NC 27599

${ }^{\mathrm{d} D e p a r t m e n t}$ of Psychiatry \& Behavioral Sciences, Duke University Medical Center, 238 Civitan Building, Box 3173, Durham, NC 27710

\section{Abstract \\ Objective-The primary aim is to describe drug and alcohol trajectories in adults with schizophrenia.}

Method-Growth mixture models were used to examine disordered and non-disordered use and abstinence in the Clinical Antipsychotic Trials of Intervention Effectiveness trial.

Results-Five classes—always abstinent; fluctuating use, abuse, and occasional abstinence; occasional (ab)use; stopped (ab)use; abusing - fit best. Overlap exists between always abstinent drug and alcohol classes; less overlap exists across other classes.

Conclusion-There is heterogeneity in drug and alcohol use among adults with schizophrenia. The lack of overlap between classes, save always abstinent, suggests modeling drug and alcohol use separately.

(C) 2013 Elsevier B.V. All rights reserved.

"Corresponding author: Richard Van Dorn, RTI International, 3040 E. Cornwallis Road, P.O. Box 12194, Research Triangle Park, NC 27709, Phone: (919) 541-6724, Fax: (919) 485-5555, rvandorn@ rti.org.

Disclosure

The content is solely the responsibility of the authors and does not necessarily represent the official views of the NIDA, the NIMH, or the NIH.

Conflict of interest

The authors report no conflicts of interest in connection with any aspect of the research reported in this article.

Contributors

Proposed the study: RAVD, SLD. Managed the literature searches: KLJ. Analyzed the data: ST. Interpretation of findings: RAVD, SLD, ST, JMJ, KLJ, MSS. Wrote the paper: RAVD, SLD, ST. Revised the paper: RAVD, SLD, ST, JMJ, KLJ, MSS. All authors contributed to and have approved the final manuscript.

Publisher's Disclaimer: This is a PDF file of an unedited manuscript that has been accepted for publication. As a service to our customers we are providing this early version of the manuscript. The manuscript will undergo copyediting, typesetting, and review of the resulting proof before it is published in its final citable form. Please note that during the production process errors may be discovered which could affect the content, and all legal disclaimers that apply to the journal pertain. 


\section{Keywords}

drug use; alcohol use; schizophrenia; longitudinal; growth mixture model

\section{Introduction}

Substance use is overrepresented in adults with schizophrenia compared to the general population (Regier et al., 1990) and increases risk for poor clinical, health, social, and legal outcomes (Swartz et al., 2006). Despite its high prevalence and deleterious consequences, substance use remains one of the most vexing clinical complications in this population. This, in part, may be due to the limited application of advanced statistical approaches, such as growth mixture modeling (GMM), that describe patterns of substance use over time.

There have been few applications of GMM to explore substance use trajectories in adults with mental illness (Xie et al., 2006; Xie et al., 2009). A few studies have applied GMM to examine psychiatric trajectories among adults with mental illness (Chi and Weisner, 2008) and medication response in adults with schizophrenia (Marques et al., 2011; Muthén and Brown, 2009). Additionally, studies that have utilized GMMs to investigate substance use have focused on disordered use or remission as opposed to including non-disordered use. Prior studies also have focused on patients with diagnosed, co-occurring mental and substance use disorders, instead of including subjects with subtly reducing or escalating patterns. Moreover, drug and alcohol trajectories have not been examined separately, though important differences may exist. Finally, measurement periods have been large, ranging from six weeks to four years. This study adds to the extant literature by examining drug and alcohol trajectories using GMMs and horizontal line plots (Tueller, 2013) in a large sample of adults with schizophrenia.

\section{Method}

\subsection{Sample}

Data are from the Clinical Antipsychotic Trials of Intervention Effectiveness (CATIE) study, a randomized clinical trial investigating the cost-effectiveness of atypical and conventional antipsychotic medications $(N=1460)$. The CATIE study was conducted at over 50 U.S. sites. Only $7 \%$ of screened patients were excluded, and the sample resembled a usual-care, noninterventional sample (Swanson et al., 2006). Study design and entry criteria are presented elsewhere (Stroup et al., 2003). The CATIE protocol was approved by local IRBs, and participants gave written informed consent. The RTI International IRB approved the current study's protocol.

\subsection{Method}

2.2.1. Substance use-The CATIE study used a multi-modal approach to assess alcohol and drug use: (a) SCIDs (First et al., 1996) completed at baseline by MA-level clinicians to diagnose past month substance use disorders; (b) Alcohol Use Scale and Drug Use Scale ratings (Drake et al., 1996) completed by an MD or other clinician regarding patients' substance use during the prior three months; (c) participants' self-reported alcohol and drug use during the prior three months; (d) family members/caregivers' ratings of participants' problems with excessive drug or alcohol use in the prior month; and (e) biological tests (hair radioimmunoassay and urinalysis for drug use). Results were used to determine alcohol and drug abuse/dependence, non-disordered use, and abstinence at each assessment as described in Van Dorn et al. (2012a) and Desmarais et al. (2012). 
2.2.2. Procedures-The CATIE study included baseline, $1,3,6,9,12,15$, and 18 month and end-of-phase assessments.

2.2.3. Analysis-We utilized linear multilevel (clustered by study site) GMMs to examine intra-individual drug and alcohol trajectories using Mplus 7. GMMs were used to extend the estimation of inter-individual variation around one average latent growth curve that describes the intra-individual rate of change in substance use across time. A maximumlikelihood approach was used to classify participants into different latent trajectory classes, each representing a different unobserved subpopulation with its own average latent growth curve. Latent class membership for each participant was estimated by maximizing the posterior probability of group membership (Muthén, 2001, 2004; Muthén and Shedden, 1999). The Bayesian Information Criterion was used to compare GMMs with different numbers of classes (Nylund et al., 2007). ${ }^{1}$ The first class (i.e., always abstinent) was fixed a priori using the training data option. Within class parameters were constrained to reflect this lack of variation. All other participants were allowed to be assigned to the first or any other class.

Missing data were addressed using maximum likelihood estimation assuming missingness at random (MAR), meaning that missingness assumptions hold after controlling for the variables that were related to missingness in the model (Enders, 2010). Exploratory analyses suggested that baseline drug and alcohol values were more strongly predictive of study attrition than other variables. Because baseline values were part of the model, we assumed MAR. All cases with at least one observation were included. In each GMM, class membership was regressed on age, education, sex, minority racial status, and any hospitalization three months prior to enrollment.

\section{Results}

Most participants were male (73.9\%; $n=1,079)$, white $(60 \% ; n=874)$, not married nor cohabitating $(81.0 \%, n=1,181)$, and had completed high school $(74.3 \%, n=1,085)$. At baseline, average age was 40.56 years $(S D=11.10$, range $18-65)$ and $39.7 \%(n=579)$ were not using, while $60.3 \%$ ( $n=881)$ reported some substance use.

Five-class models fit best for both drugs and alcohol. Participants were assigned to the class for which they had the greatest posterior probability of belonging (Lubke and Tueller, 2010). Figure 1 contains horizontal line plots of participants' drug and alcohol trajectories stratified by latent class. White, orange, and red lines designate abstinence, non-disordered use, and abuse/dependence, respectively. Latent classes and sample sizes within each class were always abstinent (class 1, drug: $n=701$, alcohol: $n=627$ ); fluctuating use, abuse, and occasional abstinence (class 2, drug: $n=285$, alcohol: $n=356$ ); occasional (ab)use (class 3 , drug: $n=307$, alcohol: $n=198$ ); stopped (ab)use (class 4, drug: $n=62$, alcohol: $n=220$ ); and abusing (class 5, drug: $n=102$, alcohol: $n=56$ ). Note that drug users who stopped are in class 3 instead of 4, while alcohol (ab)users who stopped are in the class 4. Variances of the random intercepts and slopes were smaller in the alcohol models than drug models indicating greater individual differences in drug than alcohol trajectories.

There was considerable agreement between membership in the always abstinent drug and alcohol classes: $65.2 \%$ of those who always abstained from drugs also always abstained from alcohol. The next highest level of agreement was between the second classes: $51.6 \%$ of those in the fluctuating use, abuse, and occasional abstinence drug class were in the same

\footnotetext{
${ }^{1}$ The bootstrap likelihood ratio tests for testing k vs. k-1 classes are not available when using the training data option in Mplus.
} 
alcohol class. The greatest discrepancy was found between stopped (ab)use classes: Only $11.8 \%$ of those who stopped alcohol (ab)use also stopped drug (ab)use.

Table 1 displays odds ratios (ORs) from regressing class on baseline factors within the GMMs; using baseline factors to predict class membership in the GMM helps to profile the unobserved subpopulation represented by each class. Rows contain the target class; columns contain the reference class. Using drug classes and hospitalization as an example, cases in class 2 were 2.59 times more likely to have been hospitalized in the 3 months prior to the baseline assessment than cases in class 1 . Overall, the large number of statistically significant $O R$ s highlights the differences in the likelihood of membership among the five classes by age, education, sex, minority racial status, and pre-study hospitalization.

\section{Discussion}

We report new findings from the CATIE study, comparing drug and alcohol trajectories for adults with schizophrenia identified using GMMs. This represents the first examination of substance use trajectories in this population. Five latent classes describe patterns of drug and alcohol use best in these patients: always abstinent; fluctuating use, abuse, and occasional abstinence; occasional (ab)use; stopped (ab)use; and abusing. There is substantial overlap between drug and alcohol always abstinent classes; however, overlap between other drug and alcohol classes was lower. Finally, baseline factors differ between classes as evidenced by the statistically significant odds ratios in Table 1 describing the differences in the likelihood of latent class membership by age, education, sex, minority racial status, and prestudy hospitalization.

Consistent with prior applications of GMM used to examine substance use in other samples (e.g., adolescents, non-clinical adults) (Chassin et al., 2004), the multiple classes indicate heterogeneity in substance use over time among adults with schizophrenia. This finding has important implications for research and practice. First, prior research on substance use among adults with schizophrenia has assumed homogeneity. Our findings suggest this assumption is inappropriate and support the need for approaches, such as GMM that model heterogeneity. Second, there was some within-class heterogeneity (see Figure 1), as seen by changes in the patterns of abstinence, use, and disordered use over time, indicating the need for ongoing, repeated assessments. Third, the lack of overlap between drug and alcohol classes, with the exception of always abstinent, as well as differential outcomes associated with drug and alcohol use (Van Dorn et al., 2012b), supports modeling drugs and alcohol separately.

With regard to clinical implications, our findings contribute to a literature supporting multiple, distinct substance use trajectories, suggesting the need to tailor treatment to individual patients. As noted above, some patients' substance use fluctuated (i.e., fluctuating use, abuse, and occasional abstinence; occasional (ab)use; and stopped (ab)use) whereas others demonstrated more stability (i.e., always abstinent and abusing). Treatments targeting individual patterns of use should be most effective, but require ongoing (re)assessment of use. Findings also support a harm reduction approach that allows for fluctuations in substance use, as well as consideration of readiness for change, towards the ultimate goal of abstinence (Kerfoot et al., 2011). For example, for those who transitioned to sustained abstinence, there were some fluctuations in use prior to sustained abstinence. Finally, research suggests that integrated treatment for psychiatric and substance use problems will improve outcomes for patients who demonstrate ongoing use (i.e., fluctuating use, abuse, and occasional abstinence; occasional (ab)use; and abusing) (Drake et al., 1998).

Overall, our findings suggest multiple trajectories of drug and alcohol use among adults with schizophrenia, as well as some overlap but also discrepancies between substance use classes. 
Generalizability should be tested against other samples of adults with schizophrenia, as well as other mental illnesses. Future research also should explore heterogeneity in treatment response and other distal outcomes based on drug and alcohol trajectories among adults with schizophrenia (Xie et al., 2010). Growth mixture models represent one way to approach these important tasks.

\section{Acknowledgments}

Within the past year, Dr. Van Dorn has received research support from NIMH, NIDA, the Department of Defense, the Stanley Foundation, the Bristol Myers Squibb Foundation, Ortho McNeil Janssen Scientific Affairs, LLC., Florida's Agency for Health Care Administration, and Florida's Department of Children and Families. Dr. Desmarais has received research support from NIMH, NIDA, the Council of State Governments Justice Division, the Department of Defense, the Department of Veterans Affairs, the Bristol Myers Squibb Foundation, Florida's Agency for Health Care Administration, and Florida's Department of Children and Families. Dr. Tueller has received research support from NIDA, the Department of Health and Human Services, the Substance Abuse and Mental Health Services Administration, National Institute of Child Health and Human Development, the Department of Defense, the Office of Naval Research, the Veterans Administration, the Department of Justice, the Office of Juvenile Justice and Delinquency Prevention, and the University of Florida. Dr. Jolley has received research support from NIMH. Dr. Swartz is a consultant to Cognitive Neurosciences Inc. and Novartis.

This paper was based on results from the Clinical Antipsychotic Trials of Intervention Effectiveness project, supported with Federal funds from the National Institute of Mental Health under contract NO1 MH90001. The aim of this project was to examine the comparative effectiveness of antipsychotic drugs in conditions for which their use is clinically indicated, including schizophrenia and Alzheimer's disease. The project was carried out by principal investigators from the University of North Carolina, Duke University, the University of Southern California, the University of Rochester, and Yale University in association with Quintiles, Inc.; the program staff of the Division of Interventions and Services Research of the NIMH; and investigators from 56 sites in the United States (CATIE Study Investigators Group). AstraZeneca Pharmaceuticals LP, Bristol-Myers Squibb Company, Forest Pharmaceuticals, Inc., Janssen Pharmaceutica Products, L.P., Eli Lilly and Company, Otsuka Pharmaceutical Co., Ltd., Pfizer Inc., and Zenith Goldline Pharmaceuticals, Inc., provided medications for the studies. This work was also supported by the Foundation of Hope of Raleigh, NC. CATIE Study Investigators Group include: Lawrence Adler, MD, Clinical Insights; Mohammed Bari, MD, Synergy Clinical Research; Irving Belz, MD, TriCounty/MHMR; Raymond Bland, MD, SIU School of Medicine; Thomas Blocher, MD, MHMRA of Harris County; Brent Bolyard, MD, Cox North Hospital; Alan Buffenstein, MD, The Queen's Medical Center; John Burruss, MD, Baylor College of Medicine; Matthew Byerly, MD, University of Texas Southwestern Medical Center at Dallas; Jose Canive, MD, Albuquerque VA Medical Center; Stanley Caroff, MD, Behavioral Health Service; Charles Casat, MD, Behavioral Health Center; Eugenio Chavez-Rice, MD, El Paso Community MHMR Center; John Csernansky, MD, Washington University School of Medicine; Pedro Delgado, MD, University Hospitals of Cleveland; Richard Douyon, MD, VA Medical Center; Cyril D'Souza, MD, Connecticut Mental Health Center; Ira Glick, MD, Stanford University School of Medicine; Donald Goff, MD, Massachusetts General Hospital; Silvia Gratz, MD, Eastern Pennsylvania Psychiatric Institute; George T. Grossberg, MD, St. Louis University School of Medicine- Wohl Institute; Mahlon Hale, MD, New Britain General Hospital; Mark Hamner, MD, Medical University of South Carolina and Veterans Affairs Medical Center; Richard Jaffe, MD, Belmont Center for Comprehensive Treatment; Dilip Jeste, MD, University of California-San Diego, VA Medical Center; Anita Kablinger, MD, Louisiana State University Health Sciences Center; Ahsan Khan, MD, Psychiatric Research Institute; Steven Lamberti, MD, University of Rochester Medical Center; Michael T. Levy, MD, PC, Staten Island University Hospital; Jeffrey Lieberman, MD, University of North Carolina at Chapel Hill; Gerald Maguire, MD, University of California Irvine; Theo Manschreck, MD, Corrigan Mental Health Center; Joseph McEvoy, MD, Duke University Medical Center; Mark McGee, MD, Appalachian Psychiatric Healthcare System; Herbert Meltzer, MD, Vanderbilt University Medical Center; Alexander Miller, MD, University of Texas Health Science Center at San Antonio; Del D. Miller, MD, University of Iowa; Henry Nasrallah, MD, University of Cincinnati Medical Center; Charles Nemeroff, MD, PhD, Emory University School of Medicine; Stephen Olson, MD, University of Minnesota Medical School; Gregory F. Oxenkrug, MD, St. Elizabeth's Medical Center; Jayendra Patel, MD, University of Mass Health Care; Frederick Reimherr, MD, University of Utah Medical Center; Silvana Riggio, MD, Mount Sinai Medical Center-Bronx VA Medical Center; Samuel Risch, MD, University of California-San Francisco; Bruce Saltz, MD, Henderson Mental Health Center; Thomas Simpatico, MD, Northwestern University; George Simpson, MD, University of Southern California Medical Center; Michael Smith, MD, Harbor - UCLA Medical Center; Roger Sommi, PharmD, University of Missouri; Richard M. Steinbook, MD, University of Miami School of Medicine; Michael Stevens, MD, Valley Mental Health; Andre Tapp, MD, VA Puget Sound Health Care System; Rafael Torres, MD, University of Mississippi; Peter Weiden, MD, SUNY Downstate Medical Center; James Wolberg, MD, Mount Sinai Medical Center.

Funding for this study was provided by NIDA Award Number 1R03DA030850 to Dr. Van Dorn and supported Dr. Van Dorn's and Dr. Tueller's work on this study. Dr. Desmarais' work on this study was supported by NIDA 
Award Number P30DA028807. Dr. Jolley's work on this study was supported by the National Research Service Award Postdoctoral Traineeship from the NIMH (T32MH19117) sponsored by Cecil G. Sheps Center for Health

Services Research, University of North Carolina at Chapel Hill.

\section{References}

Chassin L, Flora DB, King KM. Trajectories of alcohol and drug use and dependence from adolescence to adulthood: the effects of familial alcoholism and personality. J Abnorm Psychol. 2004; 113(4):483-498. [PubMed: 15535782]

Chi FW, Weisner CM. Nine-Year Psychiatric Trajectories and Substance Use Outcomes: An Application of the Group-Based Modeling Approach. Eval Rev. 2008; 32(1):39-58. [PubMed: 18198170]

Desmarais SL, Van Dorn RA, Sellers BG, Young MS, Swartz MS. Accuracy of self-report, biological tests, collateral reports and clinician ratings in identifying substance use disorders among adults with schizophrenia. Psychology of Addictive Behaviors Advance online publication. 201210.1037/ a0031256

Drake RE, Mercer-McFadden C, Mueser KT, McHugo GJ, Bond GR. Review of integrated mental health and substance abuse treatment for patients with dual disorders. Schizophrenia Bulletin. 1998; 24(4):589-608. [PubMed: 9853791]

Drake, RE.; Mueser, KT.; McHugo, GJ. Clinical rating scales: Alcohol Use Scale (AUS), Drug Use Scale (DUS), and Substance Abuse Treatment Scale (SATS). In: Sederer, LI.; Dickey, B., editors. Outcomes assessment in clinical practice. Williams \& Wilkins; Baltimore, MD: 1996. p. 113-116.

Enders, CK. Applied missing data analysis. The Guilford Press; 2010.

First, MB.; Spitzer, RL.; Gibbon, M.; Williams, JBW. Structured Clinical Interview for Axes I and II DSM-IV Disorders-Patient Edition (SCID-I/P). Biometrics Research Department, New York State Psychiatric Institute; New York: 1996.

Kerfoot KE, Rosenheck RA, Petrakis IL, Swartz MS, Keefe RS, McEvoy JP, Stroup TS. CATIE Investigators. Substance use and schizophrenia: Adverse correlates in the CATIE study sample. Schizophrenia Research. 2011; 132(2-3):177-82. [PubMed: 21872443]

Lubke G, Tueller S. Latent class detection and class assignment: a comparison of the MAXEIG taxometric procedure and factor mixture modeling approaches. Structural Equation Modeling. 2010; 17(4):605-628.

Marques T, Arenovich T, Agid O, Sajeev G, Muthén B, Chen L, Kinon B, Kapur S. The different trajectories of antipsychotic response: antipsychotics versus placebo. Psychol Med. 2011; 41(07): 1481-1488. [PubMed: 20961479]

Muthén, B. Second-generation structural equation modeling with a combination of categorical and continuous latent variables: new opportunities for latent class-latent growth modeling. In: Collins, L.; Sayer, A., editors. New methods for the analysis of change. American Psychological Association; Washington, DC: 2001. p. 291-322.

Muthén, B. Latent variable analysis: growth mixture modeling and related techniques for longitudinal data. In: Kaplan, D., editor. Handbook of quantiative methodology for the social sciences. Sage; Thousand Oaks, CA: 2004. p. 345-368.

Muthén B, Shedden K. Finite mixture modeling with mixture outcomes using the EM algorithm. Biometrics. 1999; 55:463-469. [PubMed: 11318201]

Muthén B, Brown HC. Estimating drug effects in the presence of placebo response: causal inference using growth mixture modeling. Stat Med. 2009; 28(27):3363-3385. [PubMed: 19731223]

Nylund KL, Asparouhov T, Muthén BO. Deciding on the number of classes in latent class analysis and growth mixture modeling: A Monte Carlo simulation study. Structural Equation Modeling. 2007; 14(4):535-569.

Regier DA, Farmer ME, Rae DS, Locke BZ, Keith SJ, Judd LL, Goodwin FK. Comorbidity of mental disorders with alcohol and other drug abuse. Results from the Epidemiologic Catchment Area (ECA) Study. Journal of the American Medical Association. 1990; 264(19):2511-2518. [PubMed: 2232018]

Stroup TS, McEvoy JP, Swartz MS, Byerly MJ, Glick ID, Canive JM, McGee MF, Simpson GM, Stevens MC, Lieberman JA. The National Institute of Mental Health Clinical Antipsychotic Trials 
of Intervention Effectiveness (CATIE) project: Schizophrenia trial design and protocol development. Schizophrenia Bulletin. 2003; 29(1):15-31. [PubMed: 12908658]

Swanson JW, Swartz MS, Van Dorn RA, Elbogen EE, Wagner HR, Rosenheck RA, Stroup TS, McEvoy JP, Lieberman JA. A national study of violent behavior in persons with schizophrenia. Archives of General Psychiatry. 2006; 63(5):490-499. [PubMed: 16651506]

Swartz MS, Wagner HR, Swanson JW, Stroup TS, McEvoy JP, Canive JM, Miller DD, Reimherr F, McGee M, Kahn A, Van Dorn RA, Rosenheck RA, Lieberman JA. Substance use in persons with schizophrenia: Baseline prevalence and correlates from the NIMH CATIE study. The Journal of Nervous and Mental Disease. 2006; 194:164-172. [PubMed: 16534433]

Tueller, S. longCatEDA: Package for Plotting Categorical Longitudinal and Time-Series Data. R package version 013. 2013. http://cran.r-project.org/web/packages/longCatEDA/longCatEDA.pdf

Van Dorn RA, Desmarais SL, Scott Young M, Sellers BG, Swartz MS. Assessing illicit drug use among adults with schizophrenia. Psychiatry Res. 2012a; 200(2-3):228-236. [PubMed: 22796100]

Van Dorn RA, Volavka J, Johnson N. Mental disorder and violence: is there a relationship beyond substance use? Soc Psychiatry Psychiatr Epidemiol. 2012b; 47(3):487-503. [PubMed: 21359532]

Xie H, Drake R, McHugo G. Are there Distinctive Trajectory Groups in Substance Abuse Remission over 10 years? An Application of the Group-Based Modeling Approach. Administration and Policy in Mental Health and Mental Health Services Research. 2006; 33(4):423-432. [PubMed: 16691463]

Xie H, McHugo G, Drake R. Subtypes of clients with serious mental illness and co-occurring disorders: latent-class trajectory analysis. Psychiatric Services. 2009; 60(6):804-811. [PubMed: 19487351]

Xie H, McHugo G, He X, Drake R. Using the group-based dual trajectory model to analyze two related longitudinal outcomes. Journal of Drug Issues. 2010; 40(1):45-61. 


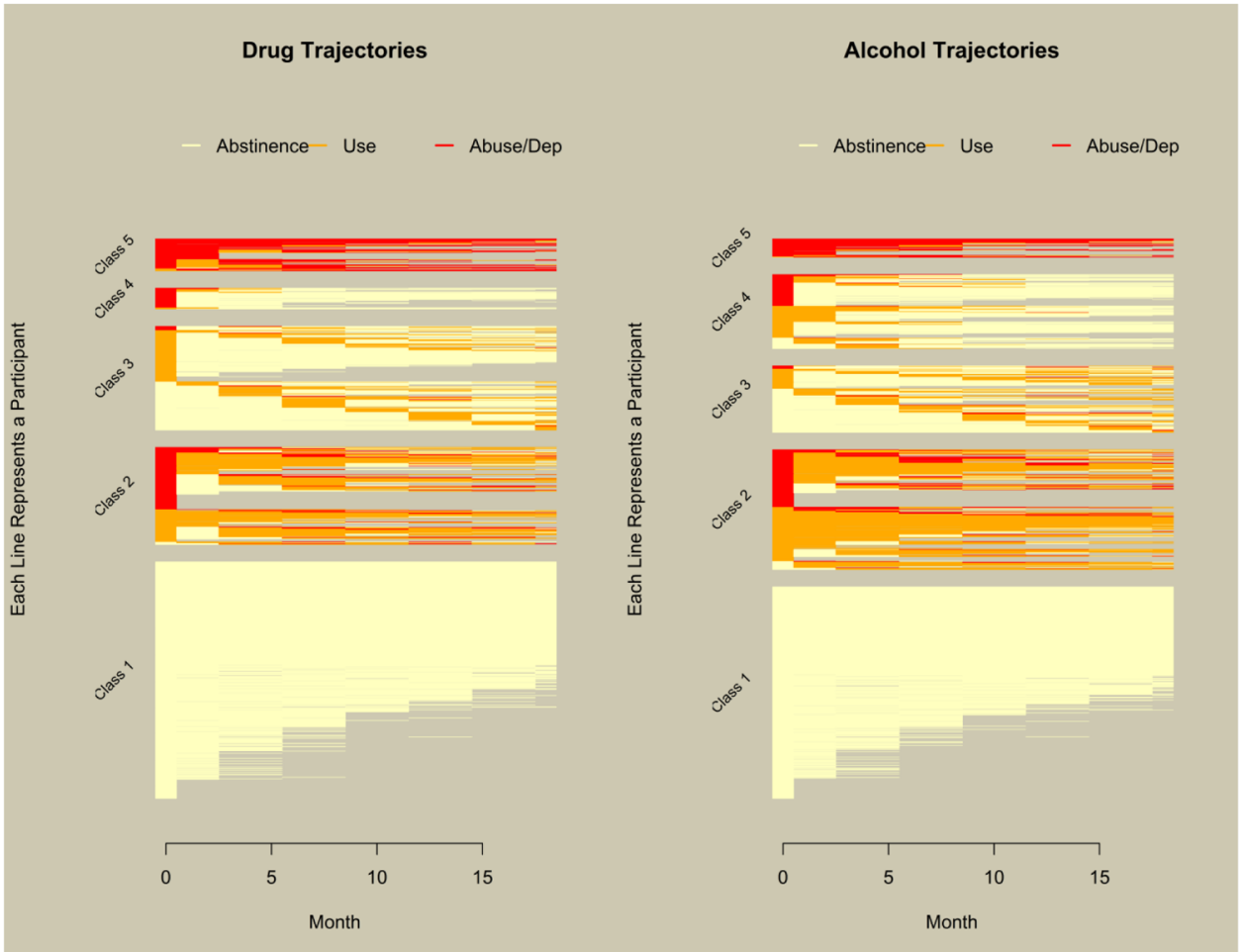

Figure 1.

Drug and alcohol trajectories plotted by latent class. 


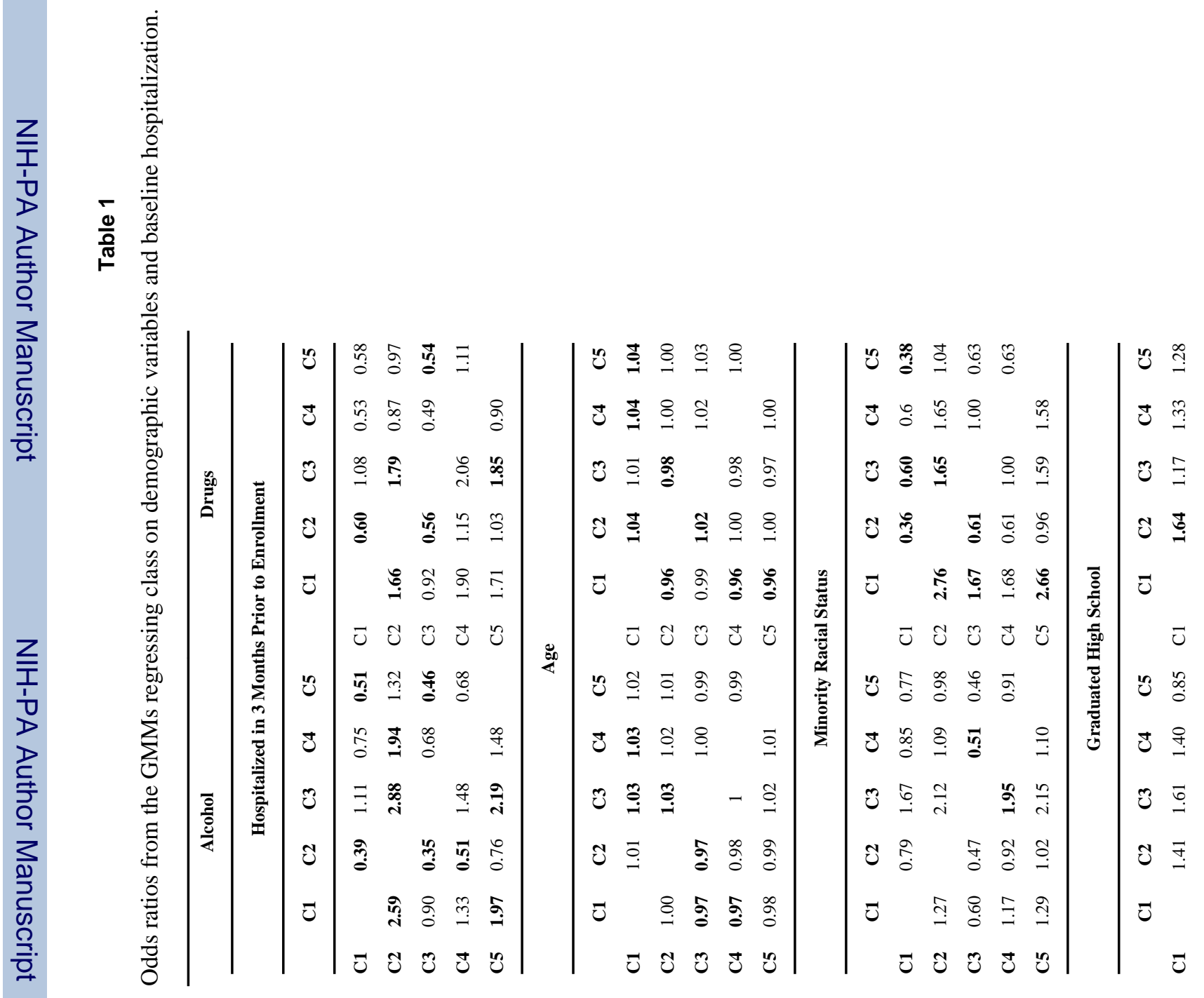




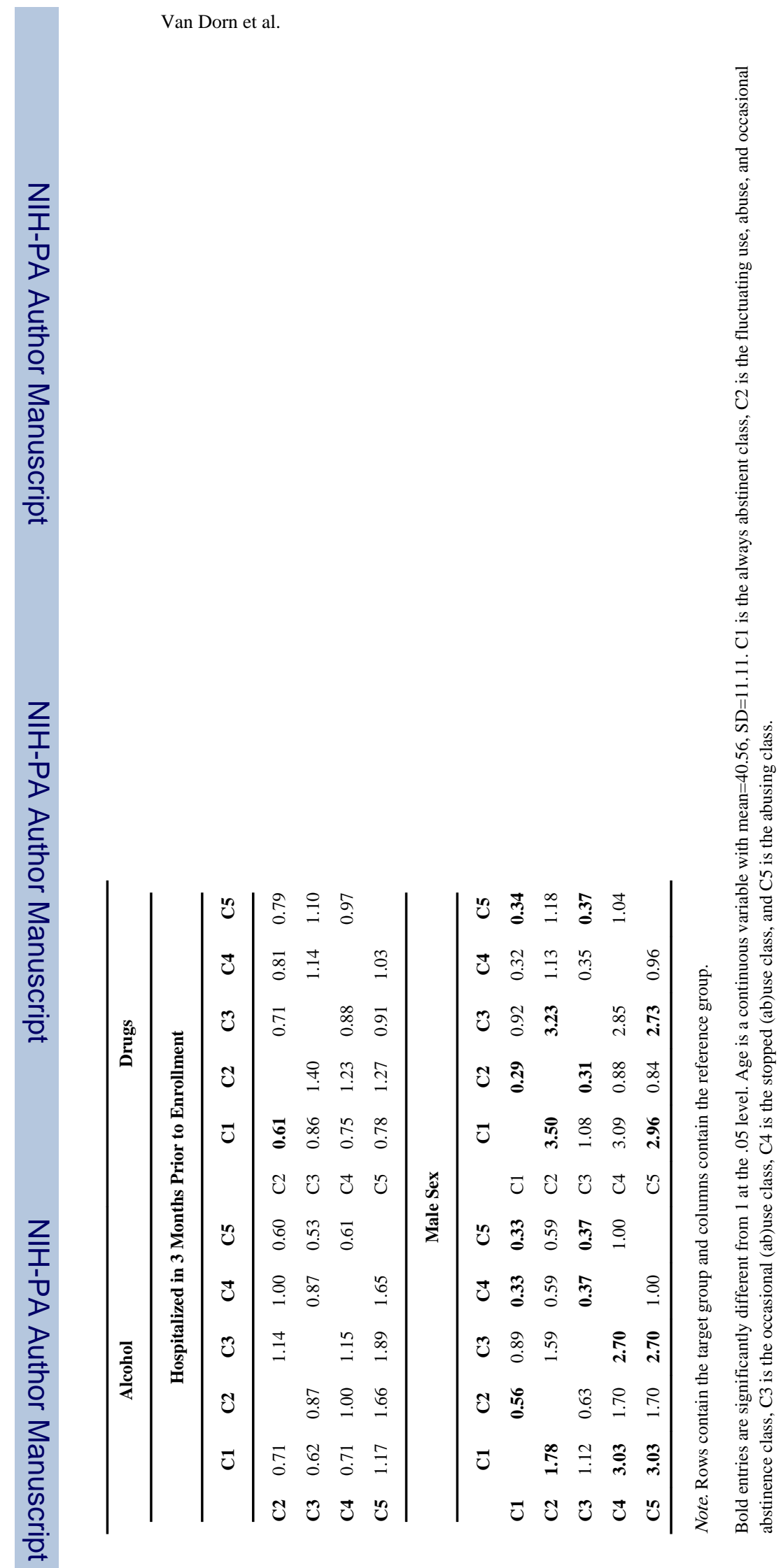

Page 10

Schizophr Res. Author manuscript; available in PMC 2014 August 01. 